\title{
Elevated Osteoprotegerin Concentration Predicts Increased Risk of Cardiovascular Mortality in Patients with Chronic Kidney Disease: A Systematic Review and Meta- Analysis
}

\author{
Qing-xiu Huang ${ }^{a}$ Jian-bo Li ${ }^{b, c}$ Naya Huang ${ }^{b, c}$ Xiao-wen Huang ${ }^{d}$ \\ Yan-lin $\mathrm{Li}^{\mathrm{a}}$ Feng-xian Huang ${ }^{\mathrm{b}, \mathrm{c}}$ \\ a Department of Nephrology, Zhongshan Hospital of Traditional Chinese Medicine, Affiliated \\ to Guangzhou University of Chinese Medicine, Zhongshan, PR China; ${ }^{b}$ Department of \\ Nephrology, The First Affiliated Hospital, Sun Yat-sen University, Guangzhou, PR China; \\ 'Key Laboratory of Nephrology, National Health Commission and Guangdong Province, \\ Guangzhou, PR China; ${ }^{d}$ Department of Ultrasonography, Zhongshan Hospital of Traditional \\ Chinese Medicine, Affiliated to Guangzhou University of Chinese Medicine, Zhongshan, \\ PR China
}

\section{Keywords}

Osteoprotegerin · Cardiovascular mortality $\cdot$ Chronic kidney disease $\cdot$ Meta-analysis

\begin{abstract}
Introduction: Studies have shown inconsistent results regarding the association between osteoprotegerin (OPG) concentration and cardiovascular mortality in patients with chronic kidney disease (CKD). This systematic review and meta-analysis aims to investigate the association between OPG concentration and cardiovascular mortality in patients with CKD. Methods: Between January 1970 and February 2020, the PubMed, EMBASE, and Cochrane Library databases were searched for eligible studies investigating the association between OPG concentration and cardiovascular mortality in patients with CKD. Pooled hazard ratios (HRs) and the corresponding $95 \%$ confidence intervals (Cls) were calculated using random effects models. Results: In total, 10 studies comprising 2,120 patients (including 1,723 receiving dialysis) with CKD were included. The included studies were considered to be of fair to high quality. Patients in the highest OPG concentration group had a significantly higher risk of cardiovascular mortality (4 studies; adjusted $\mathrm{HR}, 2.05 ; 95 \% \mathrm{Cl}, 1.39-3.00$ ) than patients in the low OPG concentra-
\end{abstract}

Qing-xiu Huang and Jian-bo Li contributed equally to this work.

Feng-xian Huang

Department of Nephrology, The First Affiliated Hospital

Sun Yat-sen University, 58th, Zhongshan Road II

Guangzhou 510080 (PR China)

hfxyl@163.net
Yan-lin Li

Department of Nephrology, Zhongshan Hospital of Traditional Chinese Medicine, Affiliated to Guangzhou University of Chinese Medicine, 3rd, Kangxin Road

Zhongshan 528400 (PR China)

li.yan.lin@126.com 


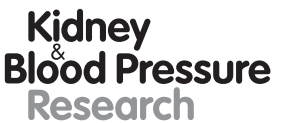

Kidney
Blood Pressure Research

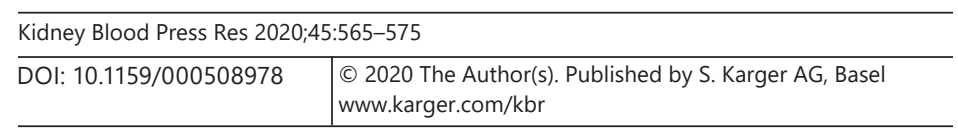

Huang et al.: OPG Predicts Cardiovascular Mortality in CKD

tion group. An increase of $1 \mathrm{pmol} / \mathrm{L}$ in OPG concentration was associated with a $4 \%$ increased risk of cardiovascular mortality (6 studies; adjusted $\mathrm{HR}, 1.04 ; 95 \% \mathrm{Cl}, 1.02-1.07$ ). Conclusion: Elevated OPG concentrations are associated with an increased risk of cardiovascular death in patients with CKD.

(C) 2020 The Author(s)

Published by S. Karger AG, Basel

\section{Introduction}

Cardiovascular mortality is the leading cause of death in patients with chronic kidney disease (CKD), and these patients have a 10-30 times higher cardiovascular mortality risk than the general population [1]. In addition to the classic tetrad of smoking, diabetes, dyslipidaemia, and hypertension, mineral and bone metabolism disorders are also identified as risk factors for cardiovascular mortality in patients with CKD [2-4]. Osteoprotegerin (OPG) is a secretory glycoprotein of the TNF receptor superfamily [5] and has been reported to be involved in bone metabolism regulation [6]. OPG inhibits osteoclastogenesis by binding to the receptor activator of nuclear factor- $\kappa B$ ligand (RANKL), which prevents RANKL from binding to the receptor activator of nuclear factor- $\kappa B$ (RANK) [7]. OPG concentrations were reported to be higher in CKD patients than in the general population [8] and have been associated with adverse cardiovascular outcomes [9-11].

Some studies have indicated that an elevated OPG concentration was an independent predictor of cardiovascular mortality in patients with CKD [11-14]. However, several studies determined that OPG was not independently associated with cardiovascular mortality in CKD patients [15-17]. A previous meta-analysis indicated that OPG was significantly associated with cardiovascular mortality (pooled hazard ratio [HR], 2.53; 95\% confidence interval [CI], 1.29-4.94) [18]. However, this meta-analysis included only 3 studies and qualitatively compared the upper tertile to the lower tertile of OPG concentrations in a subgroup of CKD patients (only haemodialysis patients) [18].

To quantitatively assess the association between OPG concentrations and cardiovascular mortality in patients with CKD, we performed a qualitative and quantitative meta-analysis of all available studies that reported the association of OPG concentration with cardiovascular mortality in patients with CKD.

\section{Methods}

\section{Literature Search}

This meta-analysis was conducted in accordance with the Preferred Reporting Items for Systematic Reviews and Meta-Analyses (PRISMA) statement and is registered with the International Prospective Register of Systematic Reviews (No. CRD42018092797) [19].

We searched for relevant studies published between January 1970 and February 2020 in the PubMed, EMBASE, and Cochrane Library databases. We used the search terms "osteoprotegerin" and "kidney". The complete search used for PubMed was as follows: "Osteoprotegerin" [MeSH] OR "Osteoprotegerin" [All Fields] OR "OPG" [All Fields] OR "OCIF Protein" [All Fields] OR "Osteoclastogenesis Inhibitory Factor" [All Fields] OR "Tumor Necrosis Factor Receptor 11b" [All Fields]) AND ("Renal Insufficiency, Chronic" [MeSH] OR "Renal" [All Fields] OR "Kidney" [All Fields] OR "Dialysis" [All Fields]). We also performed a manual search using the reference lists of key articles published in English. The search process was performed and confirmed by two investigators (Q.H. and J.L.). 


\section{Kidney \\ Blood Pressure \\ Research}

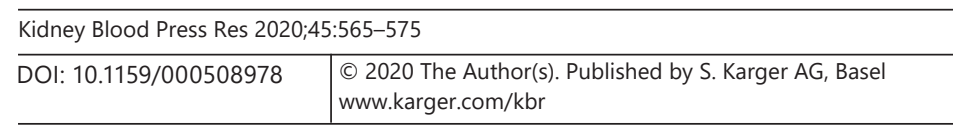

Huang et al.: OPG Predicts Cardiovascular Mortality in CKD

\section{Study Selection}

We regarded studies as eligible if they met all the following criteria: (1) OPG concentrations were measured at baseline; (2) cardiovascular death was the main outcome; (3) the studies enrolled adult patients with CKD, which was defined according to the Kidney Disease: Improving Global Outcomes (KDIGO) guidelines [20]; and (4) the studies had available data on HRs and their corresponding 95\% CIs (or provided the data needed to calculate them) for cardiovascular mortality associated with a 1-pmol/L increase in the OPG concentration, or they compared high and low OPG concentration groups. The OPG concentration groups were based on the definitions used in each study. There were no restrictions with regard to language, and published abstracts were also considered. Two reviewers (Q.H. and J.L.) independently screened the studies to select the articles. A consensus was reached through discussion with the senior author (F.H.) in cases of disagreement. Emails were sent to the corresponding authors to obtain additional data for the eligible articles if the relevant data were not reported.

\section{Data Extraction and Quality Assessment}

Two investigators (N.H. and X.H.) extracted the following data from each included study using standardized forms: author, publication year, study population, dialysis method, patient number, number of males, age of study population, OPG concentrations, follow-up duration, and the number of cardiovascular deaths. The unadjusted and most fully adjusted HRs with 95\% CIs were extracted from all the eligible studies. The quality of the studies was evaluated by consensus between the two investigators (F.H. and Y.L.) in accordance with the NewcastleOttawa Scale (NOS) (maximum score = 9) [21]. The overall study quality was defined as poor (score 0-3), fair (score 4-6), or high (score 7-9).

\section{Statistical Analysis}

The relationship between OPG concentration and cardiovascular mortality was summarized by considering OPG concentration not only as a categorical variable (comparing the high OPG concentration group to the low OPG concentration group) but also as a continuous variable (investigating the change in cardiovascular mortality for every increase of $1 \mathrm{pmol} / \mathrm{L}$ in OPG concentration). Each HR was transformed into its natural logarithm (log HR), and the variance for each log HR was calculated from its corresponding 95\% CI. Random effects models were used to obtain the pooled log HR, and the overall HR and its $95 \% \mathrm{CI}$ were calculated by exponentiation of the pooled log HR [22].

We used Stata (version 12.0) for all statistical analyses. Statistical tests were two-sided and used a significance level of $p<0.05$. We used the Cochran $Q$ test to assess heterogeneity between studies [23]. We also performed the $I^{2}$ test to assess the magnitude of the heterogeneity between studies, with values $\leq 40 \%, 40-75 \%$, and $\geq 75 \%$ indicating low, moderate, and high degrees of heterogeneity, respectively [23-25]. A sensitivity analysis was also performed using fixed effects models instead of random effects models to obtain the pooled HR for studies with low heterogeneity. In addition, a sensitivity analysis was performed to explore the impact of each individual study by deleting one study each time.

\section{Results}

Literature Search and Study Characteristics

In total, 982 non-duplicated potential studies were identified, and 10 studies [11-17, 26-28] were included in the meta-analysis (Fig. 1). The publication years of the eligible studies ranged from 2006 to 2018 . The characteristics and quality scores of the included 


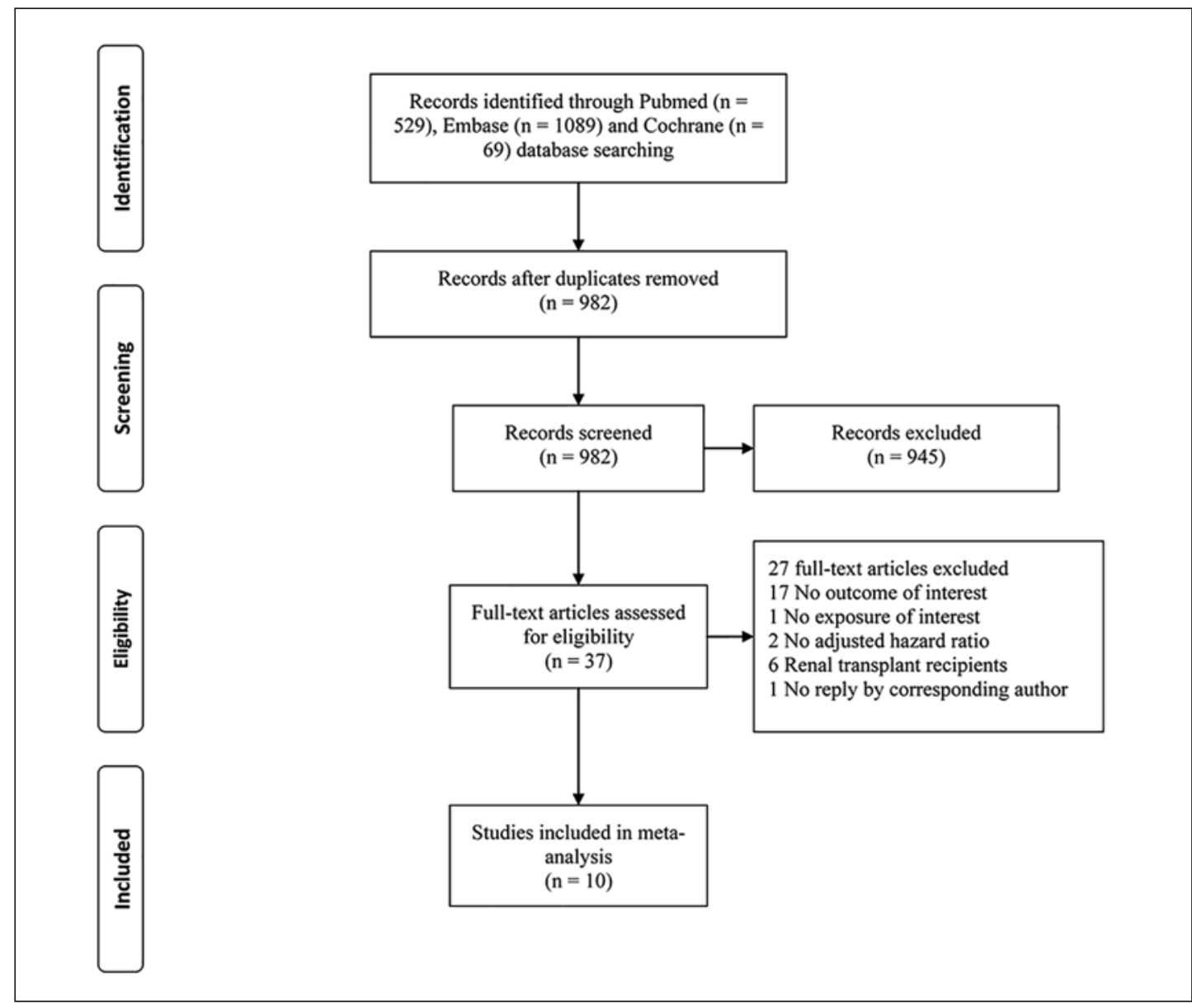

Fig. 1. Flow chart of study selection.

studies are displayed in Table 1. In total, 2,120 individuals were included. All studies were considered to be of fair (scale of 5-6) to high (scale of 7-9) quality. In total, 568 deaths were recorded. Most studies $(n=6)$ reported both unadjusted and adjusted HRs [11-14, 17, 27]; 3 studies reported only adjusted HRs $[15,16,26]$, and 1 study reported only unadjusted HRs [28].

\section{Association of OPG Concentration as a Categorical Variable with Cardiovascular Mortality}

Four studies [12,13,26,27], which included a total of 1,449 patients, reported the HR of cardiovascular mortality for the highest OPG concentration group compared to the low OPG concentration group (Fig. 2). According to the meta-analysis, patients in the highest OPG concentration group had a significantly higher risk of cardiovascular mortality (unadjusted HR, 3.63; 95\% CI, 1.97-6.72 and adjusted HR, 2.05; 95\% CI, 1.39-3.00) than patients in the low OPG concentration group. There was moderate heterogeneity for the unadjusted HR $\left(I^{2}=67.6 \%, p=0.046\right)$. After adjustment, the heterogeneity was low $\left(I^{2}=13.7 \%, p=0.324\right)$. The pooled HR did not change significantly (instrumental variable method [I-V] overall adjusted $\mathrm{HR}, 1.98 ; 95 \% \mathrm{CI}, 1.41-2.79$ ) after using the fixed effects model instead of the random effects model. 


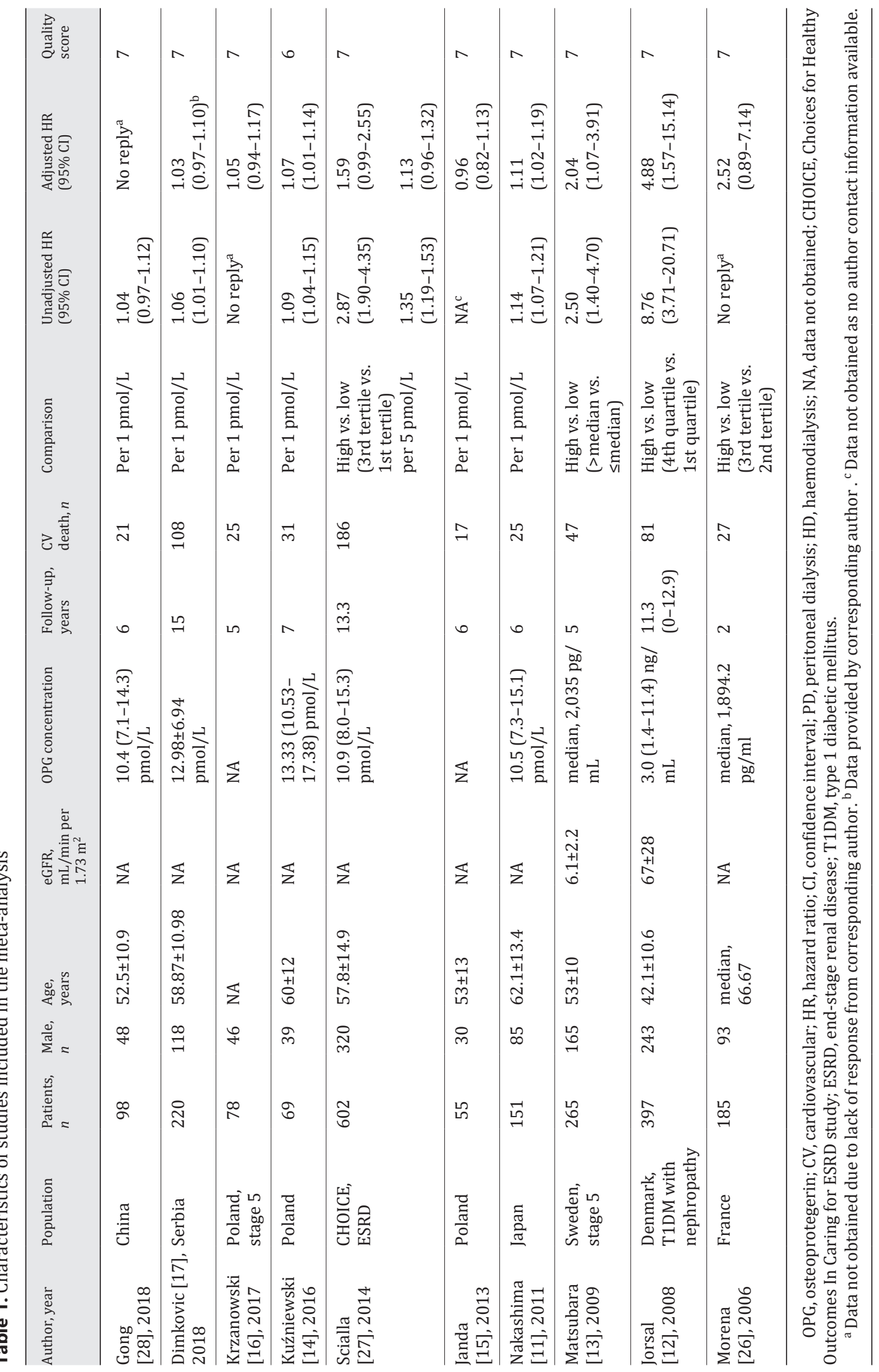




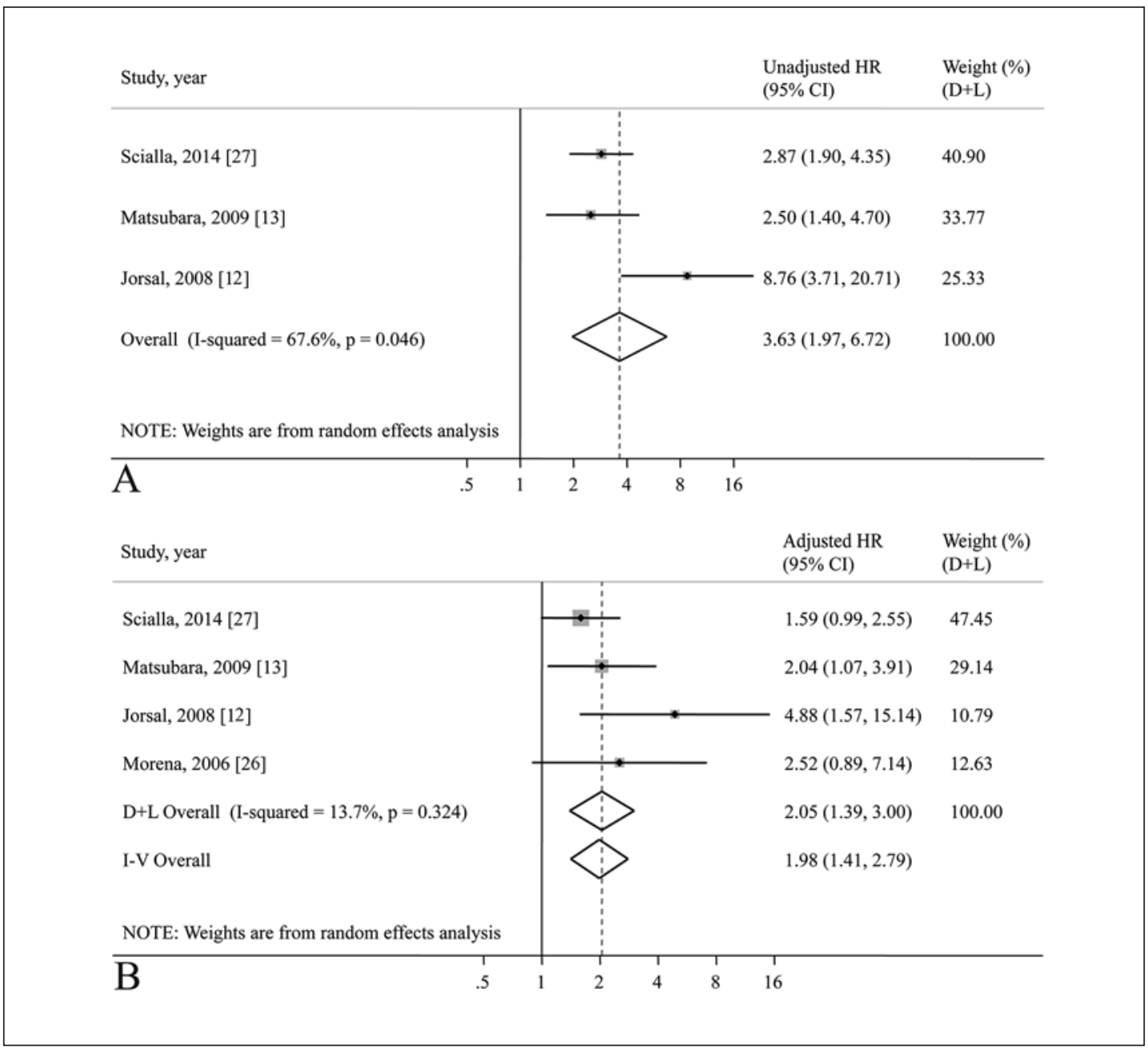

Fig. 2. Forest plot for the association of OPG concentration as a categorical variable with cardiovascular mortality. A The unadjusted HR. B The adjusted HR. D+L overall, from the random effects model; I-V overall, from the fixed effects model. D+L, DerSimonian and Laird method; I-V, instrumental variable method.

Of the 4 included studies, 1 study compared the high to low OPG concentrations according to the median value [13], 1 study compared the 3rd tertile to the 1 st tertile of OPG concentration [27], and 1 study compared the 4th tertile to the 1st tertile of OPG concentration [12]. All of the above 3 studies set the lowest OPG concentration group as the reference to investigate the association between the highest OPG concentration group and cardiovascular mortality. Only 1 study [26] set the middle OPG concentration group (2nd tertile) as the reference and found a non-significant increase in cardiovascular mortality associated with the highest OPG concentration (3rd tertile, adjusted HR, 2.52; 95\% CI, 0.89-7.14).

\section{Association of a 1-pmol/L Increase in OPG Concentration with Cardiovascular Mortality}

Seven studies [11, 14-17, 27, 28], which included a total of 1,273 patients, reported the HR of cardiovascular mortality for a 1-pmol/L increase in OPG concentration (Fig. 3). Of the 7 included studies, 1 study was published as an abstract [15], and only the adjusted HR (0.96; 95\% CI, 0.82-1.13) for the increased risk of cardiovascular mortality associated with a 1-pmol/L increase in OPG concentration was reported. Without adjustment, each 1-pmol/L 


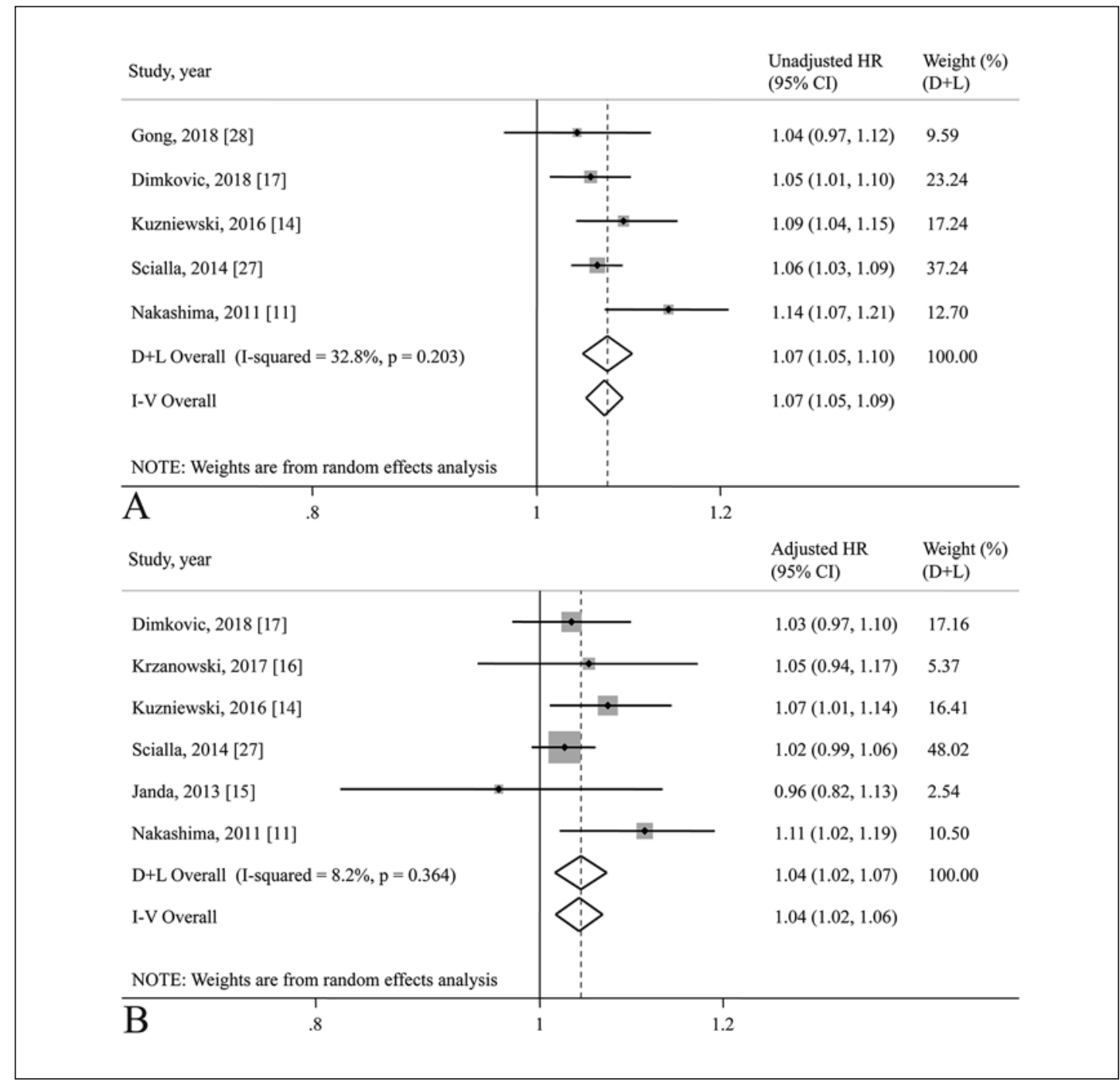

Fig. 3. Forest plot for the association of a 1-pmol/L increase in the OPG concentration with cardiovascular mortality. A The unadjusted HR. B The adjusted HR. D+L overall, from the random effects model; I-V overall, from the fixed effects model. D+L, DerSimonian and Laird method; I-V, instrumental variable method.

increase in OPG concentration was associated with a 7\% increased risk of cardiovascular mortality (unadjusted HR, 1.07; 95\% CI, 1.05-1.10), with low heterogeneity $\left(I^{2}=32.8 \%, p=\right.$ 0.203 ). The pooled HR hardly changed (I-V overall adjusted HR, 1.07; 95\% CI, 1.05-1.09) after using the fixed effects model instead of the random effects model.

After adjustment, each increase of $1 \mathrm{pmol} / \mathrm{L}$ in OPG concentration was associated with a $4 \%$ increased risk of cardiovascular mortality (adjusted HR, 1.04; 95\% CI, 1.02-1.07), with low heterogeneity $\left(I^{2}=8.2 \%, p=0.364\right)$. The pooled HR hardly changed (I-V overall, adjusted HR, 1.04; 95\% CI, 1.02-1.06) after using the fixed effects model instead of the random effects model.

\section{Sensitivity Analysis}

A sensitivity analysis was performed by removing one study at a time (Fig. 4). We found that the adjusted HR for cardiovascular mortality obtained by comparing the highest OPG 


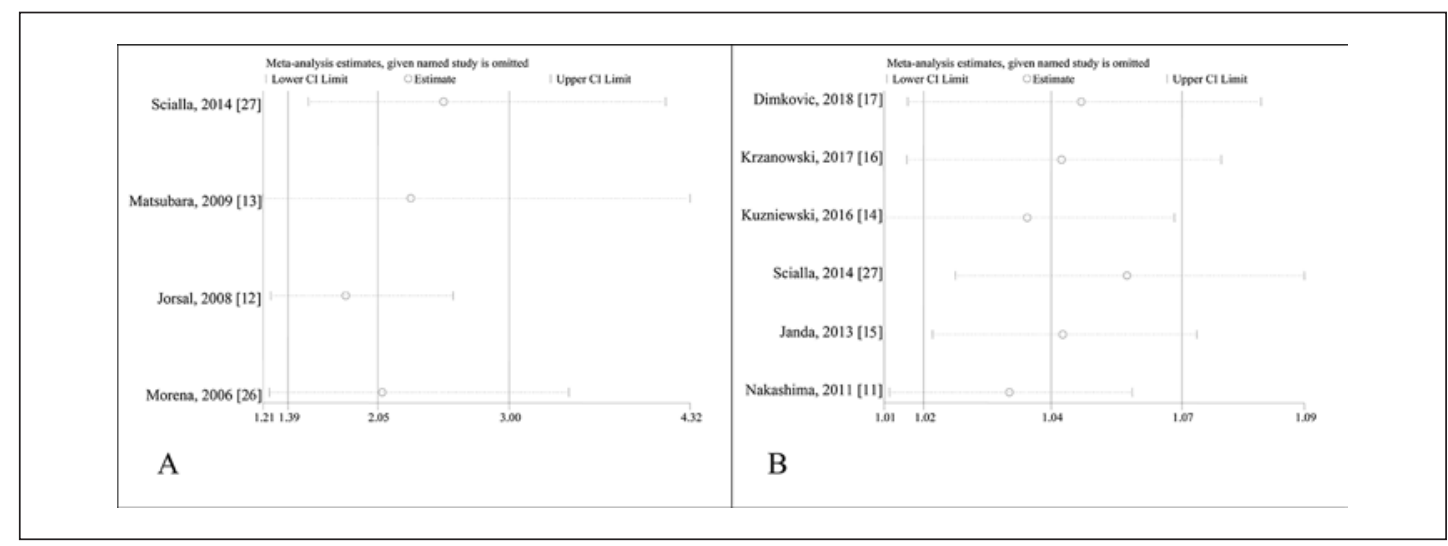

Fig. 4. Sensitivity analysis of the adjusted HRs by deleting one study each time for cardiovascular mortality comparing the highest OPG concentration group to the low OPG concentration group (A) and for cardiovascular mortality associated with each 1-pmol/L increase in OPG concentration (B).

concentration group to the low OPG concentration group was not significantly different (Fig. 4A), and neither was the adjusted HR for the change in cardiovascular mortality associated with an increase of $1 \mathrm{pmol} / \mathrm{L}$ in OPG concentration (Fig. 4B).

\section{Discussion}

The present meta-analysis investigated the association of OPG concentration with cardiovascular mortality in patients with CKD. The pooled results showed that a higher OPG concentration was associated with a higher risk of cardiovascular death in CKD patients (adjusted HR, 2.05; 95\% CI, 1.39-3.00), with low heterogeneity $\left(I^{2}=13.7 \%, p=0.324\right)$. Each increase of $1 \mathrm{pmol} / \mathrm{L}$ in OPG concentration was associated with a 4\% increased risk of cardiovascular death (adjusted HR, 1.04; 95\% CI, 1.02-1.07), with low heterogeneity $\left(I^{2}=8.2 \%, p=0.364\right)$.

The primary strength of the present meta-analysis was that the investigation of the relationship between OPG concentration and cardiovascular mortality considered OPG concentration not only as a categorical variable but also as a continuous variable. The pooled result of $\mathrm{OPG}$ as a categorical variable can just indicate qualitative judgement that higher OPG concentration suggests a higher risk of cardiovascular death, but not provide a detailed risk ratio of the higher to lower OPG concentration individual. The pooled result of OPG as a continuous variable can additionally provide the increased risk ratio of cardiovascular death by each 1-pmol/L OPG concentration increase, which can further be used to calculate the definite difference in cardiovascular death risk between patients with different OPG concentrations. Thus, OPG concentration as a continuous variable can provide an additional statistical reference value and facilitates clinical data analysis.

A previous meta-analysis also reported the predictive value of OPG to cardiovascular mortality [18]. However, this meta-analysis only included 3 studies and focused on the haemodialysis population. Our meta-analysis included more studies but still mainly focused on the dialysis (haemodialysis or peritoneal dialysis) population. Only 1 study included a nondialysis CKD population (type 1 diabetic patients with nephropathy) [12]. Therefore, there was insufficient evidence to expand the positive results between OPG and cardiovascular mortality in all CKD patients. More research studies are needed to investigate the relationship between OPG and cardiovascular mortality in the non-dialysis CKD population. 


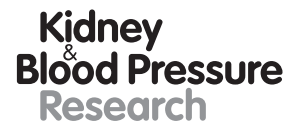

Kidney
Blood Pressure
Research

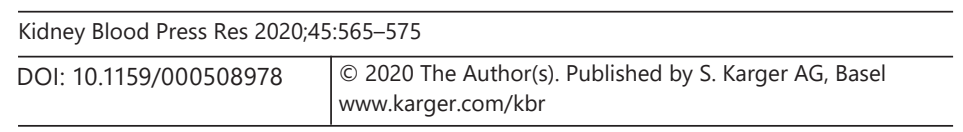

Huang et al.: OPG Predicts Cardiovascular Mortality in CKD

Elevated OPG levels may correlate with increased burden of vascular calcification. Accelerating vascular calcification was reported as the leading cause of the increased risk of cardiovascular death associated with disordered mineral metabolism [29]. OPG is a member of the TNF- $\alpha$ receptor superfamily and is involved in mineral metabolism through osteoclastogenesis inhibition, osteoclast activation, and osteoclast-like formation [5]. Previous studies have reported a positive relationship between the OPG concentration and vascular calcification, including coronary artery calcification or aortic calcification in CKD patients [30-33]. Elevated OPG levels were also reported to be independently associated with the progression of coronary artery calcification in haemodialysis patients [34]. Multiple mechanisms may explain the association between OPG and vascular calcification. Inflammation and endothelial dysfunction may be of special interest in the context of uraemia, as inflammation is a significant risk factor for cardiovascular events and mortality in both the general and CKD populations [35]. Inflammation plays a role in the production of OPG and plays an important role in the progression and complications of atherosclerosis. Moreover, endothelial dysfunction, which is linked to inflammation, is an early pathological event in atherosclerosis [36]. In addition, a higher level of OPG likely indicates a compensatory increase in the level of OPG in the vascular wall to counteract vascular calcium deposition or may result from the transition of vascular smooth muscle cells to cells resembling osteoblasts. Together, these results are consistent with current evidence in favour of a dynamic and active process of vascular calcification contributing to the increased risk of cardiovascular mortality in CKD patients [37]. Hence, the main implication of elevated OPG activity is the promotion and progression of vascular calcification, which might explain the significant association of OPG with cardiovascular mortality.

This meta-analysis had several limitations. First, as the studies included in this metaanalysis were observational in nature, it was impossible to fully adjust for potential confounders, such as nutritional status and declining kidney function during follow-up. Second, when investigating the relationship between the OPG concentration as a categorical variable and cardiovascular mortality, each study adjusted for different factors and had varied definitions and cut-off values for the OPG concentration groups. Third, a relatively small number of studies were included in the meta-analysis. Thus, the funnel plots and Egger's test were not valid because the accuracy of these tests is low or may even be misleading when fewer than 10 studies are available for the quantitative summary [38]. Finally, selective reporting bias in the literature may have influenced the present findings.

In conclusion, the present meta-analysis found that elevated OPG concentrations are associated with an increased risk of cardiovascular death in patients with CKD. Each increase of $1 \mathrm{pmol} / \mathrm{L}$ in the OPG concentration was associated with a $4 \%$ increased risk of cardiovascular mortality. The majority of studies derive from the dialysis population, and further study in the non-dialysis CKD population may be warranted. The mechanisms linking OPG to cardiovascular mortality deserve further investigation along with the predictive performance of $\mathrm{OPG}$ as a biomarker in the clinical setting.

\section{Statement of Ethics}

This meta-analysis was approved by the Ethics Committee of Guangzhou University of Chinese Medicine. The work was conducted in accordance with the Declaration of Helsinki.

\section{Conflict of Interest Statement}

The authors have no conflicts of interest to declare. 


\section{Kidney \\ Blood Pressure Research}

\begin{tabular}{l|l}
\hline Kidney Blood Press Res 2020:45:565-575 \\
\hline DOI: 10.1159/000508978 & $\begin{array}{l}\text { @ 2020 The Author(s). Published by S. Karger AG, Basel } \\
\text { www.karger.com/kbr }\end{array}$ \\
\hline
\end{tabular}

Huang et al.: OPG Predicts Cardiovascular Mortality in CKD

\section{Funding Sources}

This work was supported by the Natural Science Foundation of China (Grant No. 81600545, 81570750, and 81870575), the Natural Science Foundation of Guangdong Province, China (Grant No. 2017A030310199), and the Natural Science Foundation of Guangdong Province, China (Grant No. 2017A030313720)

\section{Author Contributions}

Q.H., J.L., and F.H. conceived and designed the study strategy. F.H. and Y.L. contributed to the manuscript revision. Q.H. and J.L. conducted the literature search. Q.H., J.L., and F.H. selected the included studies. N.H. and X.H. extracted data from each included study. F.H. and Y.L. assessed the study quality. Q.H. prepared the tables and all figures. F.H. supervised the study and acted as an arbitrator for all possible disagreements. All authors read and approved the content.

\section{References}

1 Foley RN, Parfrey PS, Sarnak MJ. Clinical epidemiology of cardiovascular disease in chronic renal disease. Am J Kidney Dis. 1998 Nov;32(5 Suppl 3):S112-9.

2 Raggi P, Kleerekoper M. Contribution of bone and mineral abnormalities to cardiovascular disease in patients with chronic kidney disease. Clin J Am Soc Nephrol. 2008 May;3(3):836-43.

3 Kovesdy CP, Bleyer AJ, Molnar MZ, Ma JZ, Sim JJ, Cushman WC, et al. Blood pressure and mortality in U.S. veterans with chronic kidney disease: a cohort study. Ann Intern Med. 2013 Aug;159(4):233-42.

4 Nakamura K, Nakagawa H, Murakami Y, Kitamura A, Kiyama M, Sakata K, et al.; EPOCH-JAPAN research group. Smoking increases the risk of all-cause and cardiovascular mortality in patients with chronic kidney disease. Kidney Int. 2015 Nov;88(5):1144-52.

5 Simonet WS, Lacey DL, Dunstan CR, Kelley M, Chang MS, Lüthy R, et al. Osteoprotegerin: a novel secreted protein involved in the regulation of bone density. Cell. 1997 Apr;89(2):309-19.

6 Venuraju SM, Yerramasu A, Corder R, Lahiri A. Osteoprotegerin as a predictor of coronary artery disease and cardiovascular mortality and morbidity. J Am Coll Cardiol. 2010 May;55(19):2049-61.

7 Lacey DL, Timms E, Tan HL, Kelley MJ, Dunstan CR, Burgess T, et al. Osteoprotegerin ligand is a cytokine that regulates osteoclast differentiation and activation. Cell. 1998 Apr;93(2):165-76.

8 Shaarawy M, Fathy SA, Mehany NL, Hindy OW. Circulating levels of osteoprotegerin and receptor activator of NF-kappaB ligand in patients with chronic renal failure. Clin Chem Lab Med. 2007;45(11):1498-503.

9 Mesquita M, Demulder A, Damry N, Mélot C, Wittersheim E, Willems D, et al. Plasma osteoprotegerin is an independent risk factor for mortality and an early biomarker of coronary vascular calcification in chronic kidney disease. Clin Chem Lab Med. 2009;47(3):339-46.

10 Nishiura R, Fujimoto S, Sato Y, Yamada K, Hisanaga S, Hara S, et al. Elevated osteoprotegerin levels predict cardiovascular events in new hemodialysis patients. Am J Nephrol. 2009;29(3):257-63.

11 Nakashima A, Carrero JJ, Qureshi AR, Hirai T, Takasugi N, Ueno T, et al. Plasma osteoprotegerin, arterial stiffness, and mortality in normoalbuminemic Japanese hemodialysis patients. Osteoporos Int. 2011 Jun; 22(6):1695-701.

12 Jorsal A, Tarnow L, Flyvbjerg A, Parving HH, Rossing P, Rasmussen LM. Plasma osteoprotegerin levels predict cardiovascular and all-cause mortality and deterioration of kidney function in type 1 diabetic patients with nephropathy. Diabetologia. 2008 Nov;51(11):2100-7.

13 Matsubara K, Stenvinkel P, Qureshi AR, Carrero JJ, Axelsson J, Heimbürger O, et al. Inflammation modifies the association of osteoprotegerin with mortality in chronic kidney disease. J Nephrol. 2009 Nov-Dec;22(6):77482.

14 Kuźniewski M, Fedak D, Dumnicka P, Stępień E, Kuśnierz-Cabala B, Cwynar M, et al. Osteoprotegerin and osteoprotegerin/TRAIL ratio are associated with cardiovascular dysfunction and mortality among patients with renal failure. Adv Med Sci. 2016 Sep;61(2):269-75.

15 Janda K, Krzanowski M, Dumnicka P, Kusnierz-Cabala B, Sułowicz W. Calcium scoring as a non-invasive, significant predictor of mortality in dialysis patients. Nephrol Dial Transplant. 2013;28:i443.

16 Krzanowski M, Krzanowska K, Gajda M, Dumnicka P, Dziewierz A, Woziwodzka K, et al. Pentraxin 3 as a new indicator of cardiovascular-related death in patients with advanced chronic kidney disease. Pol Arch Intern Med. 2017;127(3):170-7. 

cular calcifications in hemodialysis patients: a longitudinal study. Int Urol Nephrol. 2018 May;50(5):939-46.

Pichler G, Haller MC, Kainz A, Wolf M, Redon J, Oberbauer R. Prognostic value of bone- and vascular-derived molecular biomarkers in hemodialysis and renal transplant patients: a systematic review and meta-analysis. Nephrol Dial Transplant. 2017 Sep;32(9):1566-78.

19 Moher D, Liberati A, Tetzlaff J, Altman DG; PRISMA Group. Preferred reporting items for systematic reviews and meta-analyses: the PRISMA statement. Ann Intern Med. 2009 Aug;151(4):264-9.

20 Levin A, Stevens PE, Bilous RW, Coresh J, De Francisco AL, De Jong PE, et al. Kidney disease: improving global outcomes (KDIGO) CKD workgroup. KDIGO 2012 clinical practice guideline for the evaluation and management of chronic kidney disease. Kidney Int Suppl. 2013;3(1):1-150.

21 Stang A. Critical evaluation of the Newcastle-Ottawa scale for the assessment of the quality of nonrandomized studies in meta-analyses. Eur J Epidemiol. 2010 Sep;25(9):603-5.

22 DerSimonian R, Laird N. Meta-analysis in clinical trials. Control Clin Trials. 1986 Sep;7(3):177-88.

23 Higgins JP, Thompson SG, Deeks JJ, Altman DG. Measuring inconsistency in meta-analyses. BMJ. 2003 Sep; 327(7414):557-60.

24 Higgins JP, Thompson SG. Quantifying heterogeneity in a meta-analysis. Stat Med. 2002 Jun;21(11):1539-58.

25 Higgins JPT, Green S, editors. Cochrane handbook for systematic reviews of interventions. Version 5.1.0 [updated March 2011]. The Cochrane Collaboration. 2011. Available from: https://handbook-5-1.cochrane. org/.

26 Morena M, Terrier N, Jaussent I, Leray-Moragues H, Chalabi L, Rivory JP, et al. Plasma osteoprotegerin is associated with mortality in hemodialysis patients. J Am Soc Nephrol. 2006 Jan;17(1):262-70.

27 Scialla JJ, Kao WH, Crainiceanu C, Sozio SM, Oberai PC, Shafi T, et al. Biomarkers of vascular calcification and mortality in patients with ESRD. Clin J Am Soc Nephrol. 2014 Apr;9(4):745-55.

28 Gong L, Zheng D, Yuan J, Cao L, Ni Z, Fang W. Elevated levels of serum sclerostin are linked to adverse cardiovascular outcomes in peritoneal dialysis patients. Int Urol Nephrol. 2018 May;50(5):955-61.

29 Kalpakian MA, Mehrotra R. Vascular calcification and disordered mineral metabolism in dialysis patients. Semin Dial. 2007 Mar-Apr;20(2):139-43.

30 Jono S, Ikari Y, Shioi A, Mori K, Miki T, Hara K, et al. Serum osteoprotegerin levels are associated with the presence and severity of coronary artery disease. Circulation. 2002 Sep;106(10):1192-4.

31 Nitta K, Akiba T, Uchida K, Otsubo S, Takei T, Yumura W, et al. Serum osteoprotegerin levels and the extent of vascular calcification in haemodialysis patients. Nephrol Dial Transplant. 2004 Jul;19(7):1886-9.

32 Abedin M, Omland T, Ueland T, Khera A, Aukrust P, Murphy SA, et al. Relation of osteoprotegerin to coronary calcium and aortic plaque (from the Dallas Heart Study). Am J Cardiol. 2007 Feb;99(4):513-8.

33 Morena M, Dupuy AM, Jaussent I, Vernhet H, Gahide G, Klouche K, et al. A cut-off value of plasma osteoprotegerin level may predict the presence of coronary artery calcifications in chronic kidney disease patients. Nephrol Dial Transplant. 2009 Nov;24(11):3389-97.

34 Ozkok A, Caliskan Y, Sakaci T, Erten G, Karahan G, Ozel A, et al. Osteoprotegerin/RANKL axis and progression of coronary artery calcification in hemodialysis patients. Clin J Am Soc Nephrol. 2012 Jun;7(6):965-73.

35 Bonetti PO, Lerman LO, Lerman A. Endothelial dysfunction: a marker of atherosclerotic risk. Arterioscler Thromb Vasc Biol. 2003 Feb;23(2):168-75.

36 Xiang GD, Xu L, Zhao LS, Yue L, Hou J. The relationship between plasma osteoprotegerin and endotheliumdependent arterial dilation in type 2 diabetes. Diabetes. 2006 Jul;55(7):2126-31.

37 Schlieper G, Schurgers L, Brandenburg V, Reutelingsperger C, Floege J. Vascular calcification in chronic kidney disease: an update. Nephrol Dial Transplant. 2016 Jan;31(1):31-9.

38 Lau J, Ioannidis JP, Terrin N, Schmid CH, Olkin I. The case of the misleading funnel plot. BMJ. 2006 Sep; 333(7568):597-600. 